\title{
Staphylococcal Protein A, Panton-Valentine Leukocidin and Coagulase Aggravate the Bone Loss and Bone Destruction in Osteomyelitis
}

\author{
Tao Jinn ${ }^{a, b}$ Yue liang Zhu ${ }^{b}$ Jun Li $^{b}$ Jian Shib ${ }^{b i a o ~ q i n g ~ H e ~}{ }^{b} \quad$ Jing Ding ${ }^{b}$ \\ Yong qing $\mathrm{Xu}^{\mathrm{b}}$ \\ aThe Third Military Medical University, Chongqing, 400038, PR China; bepartment of Orthopedic \\ Surgery, Kunming General Hospital Chengdu Military Command, Kunming, PR China
}

\section{Key Words}

Osteomyelitis - Staphylococcus aureus - Protein A - Panton-Valentine leukocidin • Coagulase

\begin{abstract}
Background/Aims: Osteomyelitis is a debilitating infectious disease of the bone which is predominantly caused by Staphylococcus aureus (S. aureus). The purpose of this study was to investigate the role of the $S$. aureus virulence factors, i.e. protein A (SpA), Panton-Valentine leukocidin (PVL) and coagulase (Coa) on osteomyelitis. Methods: The effect of SpA, PVL and Coa on osteoblasts was studied through the following aspects including osteoblast proliferation, apoptosis, bone formation, bone mineralization and RANK-L expression. S. aureus overexpressing PVL, SpA or Coa was constructed and used to study the role of PVL, SpA and Coa, respectively. S. aureus silencing PVL, SpA or Coa was also constructed and used for reversing verification. Osteoblast proliferation was detected by MTT tetrazolium dye reduction assay. Apoptosis was determined by Annexin V-FITC staining. The levels of pro-caspase 3, cleaved-caspase 3, pro-caspase 9 and cleaved-caspase 9 were detected by western blot. Bone formation markers including collagen I, osteopontin and osteocalcin were detected by real time RT-PCR. Alkaline phosphatase activity was measured by adding p-nitrophenyl phosphate as a phosphatase substrate. Von kossa stain and alizarin red stain were applied for determining phosphate and calcium deposition, respectively. The RANK-L expression was tested by ELISA. Results: PVL, SpA and Coa inhibited osteoblast proliferation, induced osteoblast apotosis, prohibited bone formation and mineralization and upregulated RANK-L expression. Conclusions: PVL, SpA and Coa play a critical role on bone loss and bone destruction of osteomyelitis.
\end{abstract}




\section{Cellular Physiology Cell Physiol Biochem 2013;32:322-333 \begin{tabular}{l|l} 
and Biochemistry Published onIIne: August 05, 2013 & $\begin{array}{l}\text { C) 2013 S. Karger AG, Basel } \\
\text { www.karger.com/cpb }\end{array}$ \\
\hline
\end{tabular} Jin et al.: Virulence Factors of S. Aureus Aggravate Osteomyelitis}

\section{Introduction}

Bone development and homeostasis is achieved by a strict balance between bone formation by osteoblasts and resorption by osteoclasts [1]. During the process of bone formation, alkaline phosphatase and type I collagen are expressed at the early stage, both of which are important for bone matrix deposition and mineralization [2, 3]. When fully differentiated, mature osteoblasts also produce regulators of matrix mineralisation including osteocalcin and osteopontin $[4,5]$. Osteoblasts then eventually produce receptor activator of nuclear factor (NF)- $\kappa B$ ligand (RANKL) which acts as a signal for the recruitment, proliferation and activation of osteoclasts which initiates the resorption phase [6].

Bone is a sterile organ system, however, bacteria can reach the healthy bone by direct inoculation, haematogenous spread or from a contiguous focus of infection [7]. The invasion of pathogenic bacteria to the healthy bone leads to osteomyelitis, which is associated with significant morbidity and mortality [8]. Osteomyelitis may be acute or chronic and affect joints, long bones, vertebrae and almost any other bone. It is characterised by progressive bone destruction, severe inflammation and bone neoformation $[9,10]$. Treatment of osteomyelitis includes antimicrobial therapy, debridement with management of resultant dead space and stabilization of bone [11]. However, the treatment is often unsuccessful due to the continued emergence of antibiotic-resistant strains [12]. Besides, the infected nidus that harbours sessile matrix-protected pathogens is impermeable to antibiotics [13].

A broad range of bacterial species have been isolated from osteomeylitis, among which Staphylococcus aureus (S. aureus) is the main offender [14]. S. aureus has been identified in $38 \%$ to $67 \%$ of culture-positive cases [15]. S. aureus is a gram-positive bacterium that lives as part of the normal microflora on the skin and mucous membranes of humans and animals $[16,17]$. It permanently colonizes the moist squamous epithelium of the anterior nares of approximately $20 \%$ of the human population and is transiently associated with the rest [18]. The success of $S$. aureus as an opportunistic pathogen is primarily due to its ability to produce a large number of virulence factors, including adherence factors such as staphylococcal protein $\mathrm{A}(\mathrm{SpA})$ and exoproteins such as Panton-Valentine leukocidin (PVL), coagulase (Coa), a-haemolysin, hyaluronidase and so on [19-21]. In this study, we investigated the role of SpA, PVL and Coa in osteomyelitis. A better understanding of the mechanisms leading to bone infection may help to improve treatment or develop novel therapies of osteomyelitis.

\section{Materials and Methods}

\section{Bacterial strain and culture condition}

S. aureus 6850 (ATCC 53657) was used in this study. It was grown in tryptic soy broth (TSB) at $37^{\circ} \mathrm{C}$ with shaking. Bacteria were harvested and washed by centrifugation at 15,000 g for 5 min and finally resuspended in phosphate buffered saline (PBS). S. aureus 6850 suspensions were adjusted to $1 \times 10^{9} \mathrm{cells} / \mathrm{ml}$ for all experiments. The bacterial supernatant was prepared by growing $S$. aureus 6850 in $5 \mathrm{ml} \mathrm{TSB}$ at $37^{\circ} \mathrm{C}$ with shaking for 12-14 h and pelleted for $10 \mathrm{~min}$ at $3000 \mathrm{~g}$. Supernatants were sterile-filtered through a Millex-GP filter unit (0.22 um; Millipore, Bedford, MA, USA ) and used for the experiments.

\section{Plasmid constructions}

lukF and lukS genes which coding the PVL, SpA gene and Coa gene were amplified by PCR using $S$. aureus genomic DNA as template. The PCR primers were listed in Table 1. PCR fragments were digested with $X b a I$ and Xho I, and cloned between Xba I and Xho I in pcDNA3.1(+) vector (invitrogen, Carlsbad, CA, USA) to give pcDNA3.1(+)-PVL, pcDNA3.1(+)-SpA and pcDNA3.1(+)-Coa, respectively. Plasmids were transformed into $S$. aureus 6850 by electroporation using gene pulser ${ }^{\mathrm{R}}$ II electroporation system (Bio-Rad, Hercules, CA) described as previously protocol [22].

To inactive the PVL, SpA and Coa expression, allelic replacement was used. The procedure was performed as preciously description [23, 24]. Briefly, two fragments of about $800 \mathrm{bp}$ that flanked the left side and the right side of the sequence targeted for deletion were amplified by PCR. The two fragments 
were digested by the same restriction enzyme and fused by ligation. The fused fragment was further cloned into the shuttle plasmid PMAD (invitrogen, Carlsbad, CA, USA) which contains a temperaturesensitive origin of replication and an erythromycin resistance gene [25]. The resulting plasmid was transformed into $S$. aureus 6850 by electroporation. Disruption the targeted gene was achieved by homologous recombination. Erythromycin-sensitive white colonies, which no longer contained the pMAD plasmid, were tested by PCR to confirm the gene replacement. S. aureus silencing PVL, SpA or Coa were referred to as PVL (-), SpA(-) and Coa(-), respectively.

\section{Cell culture and infection}

The mouse clonal MC3T3-E1 pre-osteoblastic cell line (ATCC, Middlesex, UK) was used in this study.
Table 1. Primers used in plasmid construction. The restriction enzyme recognition sites are underlined

\begin{tabular}{|c|c|}
\hline Primer & Sequence $\left(5^{\prime}\right.$ to $\left.3^{\prime}\right)$ \\
\hline \multirow[t]{2}{*}{ lukF+lukS - F } & CTCTCTAGATTGTTTGGTAAT \\
\hline & GAACGGGTTTTTTCG \\
\hline \multirow[t]{2}{*}{ lukF+lukS - R } & CTCCTCGAGAGTGAATGCCCT \\
\hline & TATTTAAATAATCCGCCC \\
\hline \multirow[t]{2}{*}{$S p A-F$} & GCGTCTAGAATGTTGAAAAAG \\
\hline & AAAAACATTA \\
\hline \multirow[t]{2}{*}{ SpA - R } & CCGCTCGAGTTATAGTTCGCG \\
\hline & ACGACG \\
\hline \multirow[t]{2}{*}{ Coa - F } & CGCTCTAGAAGCTTATTTACA \\
\hline & TGGGAT \\
\hline \multirow[t]{2}{*}{ Coa - R } & CCGCTCGAGTTATTTTGTTAC \\
\hline & TCTAGGC \\
\hline
\end{tabular}
It is a common cell line and is used routinely for the assessment of osteoblast function [26, 27]. The cells were maintained in Dulbecco's Modified Eagle's Medium (DMEM, Lonza, Basel, Switzerland) supplemented with $10 \%(\mathrm{v} / \mathrm{v})$ foetal bovine serum (FBS, Invitrogen, Carlsbad, CA, USA), 2\% penicillin-streptomycin solution and $1 \%$ L-glutamine (Sigma-Aldrich, St. Louis, MO, USA ) in a 5\% carbon dioxide $\left(\mathrm{CO}_{2}\right)$ atmosphere at $37^{\circ} \mathrm{C}$. The media was replaced every 3-4 days and after confluency, cells were harvested using trypsinEDTA (Sigma-Aldrich, St. Louis, MO, USA) and re-suspended in medium.

MC3T3-E1 was infected with S. aureus 6850 as previously described [28, 29]. Briefly, MC3T3-E1 was infected with a multiplicity of infection (MOI) of 100. After $3 \mathrm{~h}$ cells were washed and lysostaphin (20mg/ $\mathrm{ml}$ ) was added for $30 \mathrm{~min}$ to lyse all extracellular S. aureus 6850, then fresh culture medium was added to the cells. The washing, lysostaphin and medium exchange was repeated every 2-3 days to remove all extracellular staphylococci, which might have been released from the infected cells.

\section{MTT cell proliferation assay}

Overnight cultures of $S$. auerus were harvested, washed and fixed in $4.8 \%$ formaldehyde. S. aureus were centrifuged at 15,000 g for 5 minutes and re-suspended in a-MEM at $1 \times 10^{9}$ cells $/ \mathrm{ml}$. MC3T3-E1 cells were plated at $5 \times 10^{5}$ cells per well and incubated with fixed S. aureus $\left(1 \times 10^{9}\right)$ or comparative S. aureus supernatant at $37^{\circ} \mathrm{C}$ with $5 \% \mathrm{CO}_{2}$ for $96 \mathrm{~h}$. Cell proliferation was measured using the MTT (3-[4,5-dimethylthiazol-2yl]-2,5 diphenyltetrazolium bromide, Sigma) assay as described previously with minor modifications [30]. Briefly, The cultures were incubated with the MTT at a final concentration of $0.5 \mathrm{mg} / \mathrm{mL}$ for $3 \mathrm{~h}$ at $37^{\circ} \mathrm{C}$. MTT is cleaved by an enzyme in the respiratory chain in mitochondria if the cell is viable, thereby generating MTT formazan, a purple, highly visible product. After purple precipitate is visible, a detergent solution was added to lyse the cells and solubilize crystals of formazan. The samples were read at a wavelength of $570 \mathrm{~nm}$. All assays were performed in triplicate. Un-infected MC3T3-E1 cells were seeded and cultured as a control.

\section{Apoptosis detection}

Apoptosis was assayed using an Annexin V-FITC/PI Apoptosis Detection Kit (Sigma-Aldrich, St. Louis, MO, USA) according to the manufacturer guidelines with minor modifications as described [31]. MC3T3-E1 cells $\left(1 \times 10^{6}\right)$ treated with formaldehyde fixed S. aureus (or S. aureus overexpressing PVL, SpA or Coa or silencing PVL, SpA or Coa), or S. aureus supernatant for $24 \mathrm{~h}$ were collected, washed with PBS twice and incubated with Annexin V-FITC/PI in the dark for $15 \mathrm{~min}$ at RT. Then the cells were washed and analyzed by flow cytometry. Un-infected osteoblasts were used as negative control. Cells in the lower right quadrant represented apoptosis and in the upper right quadrant represented necrosis or post apoptotic necrosis [32].

The levels of pro-caspase 3, cleaved-caspase 3, pro-caspase 9 and cleaved-caspase 9 were detected by western blot. MC3T3-E1 cells treated with $S$. aureus (or S. aureus overexpressing PVL, SpA or Coa or silencing PVL, SpA or Coa), or S. aureus supernatant for $24 \mathrm{~h}$ were collected and lysed in RIPA buffer containing $1 \times$ protease inhibitor cocktail on ice for 10 minutes. Lysates were centrifuged at $13,000 \times \mathrm{g}$ for 


\section{Cellular Physiology Cell Physiol Biochem 2013;32:322-333 \begin{tabular}{ll|l} 
and Biochemistry & $\begin{array}{l}\text { DOI: 10.1159/000354440 } \\
\text { Published onlIne: August 05, } 2013\end{array}$ & $\begin{array}{l}\text { (c) 2013 S. Karger AG, Basel } \\
\text { www.karger.com/cpb }\end{array}$
\end{tabular} \\ Jin et al.: Virulence Factors of S. Aureus Aggravate Osteomyelitis}

20 min and the supernatants were used. Proteins were separated on a $10 \%$ sodium dodecyl-sulfate polyacrylamide (SDS-PAGE) gel and transferred to polyvinyl difluoride (PVDF) membranes (Millipore, Bedford, MA, USA) for $1 \mathrm{~h}$. After blocking, the membranes were incubated with primary antibodies (Cell Signaling, MA, USA) against pro-caspase 3 , cleaved-caspase 3 , procaspase 9 or cleaved-caspase 9 at $4^{\circ} \mathrm{C}$ over night, respectively. The membranes were washed and then incubated with horseradish peroxidase (HRP) secondary antibody. Detection of the target proteins on the membranes was performed using the ECL Western Blotting Detection Reagents (Thermo Scientific Pierce, Rockford, IL, USA). Gyceraldehyde 3-Phosphate Dehydrogenase (GAPDH) used as a loading control was also determined in each sample.

\section{Real Time RT-PCR analyses of bone formation markers expression}

The expression of bone formation markers including collagen I, osteopontin and osteocalcin were detected by real time RT-PCR. RNA was isolated from MC3T3-E1 cells treated with $S$. aureus (or S. aureus overexpressing PVL, SpA or Coa or silencing PVL, SpA or Coa), or S. aureus supernatant on days 0, 7, 14 and 21 respectively. Isolation was carried out by using Trizol (Invitrogen, Carlsbad, CA, USA). The first-strand cDNA was synthesized by using random primers, oligo primers and PrimeScript Reverse Transcriptase (Takara, Dalian, China). The mRNA levels of type I collagen, osteopontin and osteocalcin were determined by the ABI PRISM 7300 System (Applied Biosystems, Foster City, California, USA) using the QuantiTect SYBR Green PCR Kit (Qiagen, Hilden, Germany) according to the manufacturer's instructions. As endogenous controls for normalization of the amount of RNA in the reaction and for reverse transcription efficiency, the cDNA of GAPDH was measured in parallel separate amplification reactions. Negative controls (blanks, no RNA) were included to discard contamination. The final results were determined from three independent assays. The relative quantification of target RNA was achieved by the comparative threshold cycle $\left(\mathrm{C}_{\mathrm{T}}\right)$ method and the target $\mathrm{C}_{\mathrm{T}}$ number were normalized to GAPDH. Primers for real time RT-PCR were listed in Table 2.

\section{Measurement of alkaline phosphatase activity}

Alkaline phosphatase (ALP) activity was measured by adding p-nitrophenyl phosphate (pNPP) as a phosphatase substrate which turns yellow when dephosphorylated by ALP. Briefly, after 7-day infection, MC3T3-E1 cells were lysed with lysis buffer containing 0.1 M Na acetate, 2\% Triton X-100 and 10mM pNPP and incubated in the dark for $1 \mathrm{~h}$ at $37^{\circ} \mathrm{C}$. The reaction was stopped using $0.3 \mathrm{M} \mathrm{NaOH}$ (Sigma-Aldrich, St. Louis, MO, USA) and read at a wavelength of $540 \mathrm{~nm}$. All assays were performed in triplicate.

\section{Staining the phosphates and calcium-rich deposits on MC3T3-E1}

MC3T3-E1 cells were grown on cover slips in a six-well plate. von kossa stain and alizarin red stain were applied for determining phosphate and calcium deposition, respectively. Phosphate deposition was detected after 7-day infection, while calcium deposition was detected after 21-day infection. For von kossa stain, MC3T3-E1 cells were fixed with 4\% paraformaldehyde, washed by PBS and further treated with 5\% silver nitrate solution in the dark at $37^{\circ} \mathrm{C}$ for 30 minutes. Silver nitrate solution was then completely washed away and the cells were exposed to a bright light for 15 min to develop color. For alizarin red stain, after fixation, MC3T3-E1 cells were incubated with 2\% alizarin red S stain solution for 30 min at RT and then the dye was completely washed away. The cover slips were analyzed using $200 \times$ bright field microscopy. Staining was quantified by leeching the cells of the dye and reading absorbance at $540 \mathrm{~nm}$.

Quantification of RANK-L

MC3T3-E1 cells $\left(1 \times 10^{6}\right.$ cells/well) were seeded on six well plates and incubated with $S$. aureus (or $S$. aureus overexpressing PVL, SpA or Coa or silencing PVL, SpA or Coa), or S. aureus supernatant for 24 hours. 
Fig. 1. S. aureus inhibits osteoblast proliferation. The proliferation of MC3T3-E1 cells treated with S. aureus (or S. aureus overexpressing PVL, SpA or Coa or silencing PVL, SpA or Coa), or supernatant was detected using the MTT assay at $0 \mathrm{~h}, 24 \mathrm{~h}, 48 \mathrm{~h}, 72 \mathrm{~h}$ and $96 \mathrm{~h}$. All values are the means \pm SD of three replicates, ${ }^{* *} p<0.01$ vs uninfected.

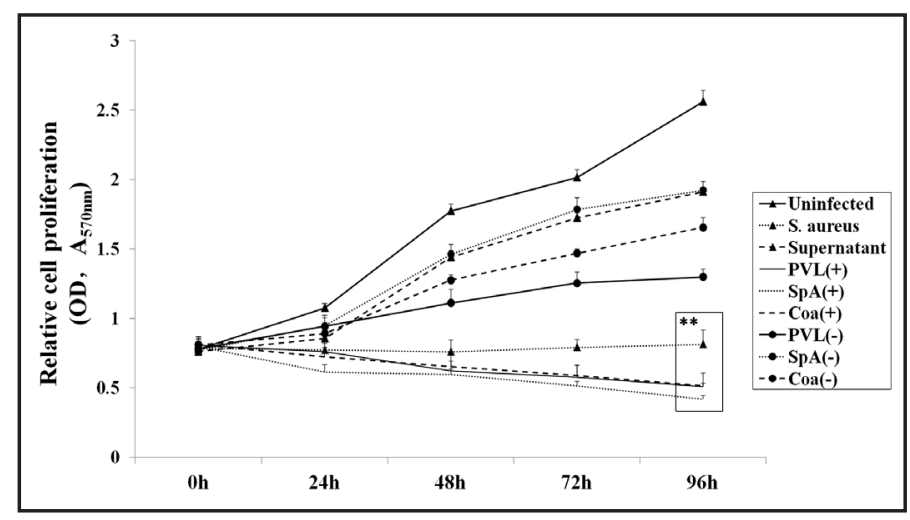

Then the culture media was removed, the cells were lysed in $200 \mathrm{ml}$ RIPA buffer. The lysate was centrifuged at 10,000 $\mathrm{g}$ for 2 minutes at $4^{\circ} \mathrm{C}$. RANK-L was detected using an ELISA kit (R\&D Systems, Minneapolis, MN, USA) according to manufacturer's instructions.

\section{Statistical analysis}

All data were expressed as means \pm SD from triplicate experiments performed in a parallel manner unless otherwise indicated. ANOVA with $F$ test or Scheffe's post-hoc multiple comparison test was used to determine statistical significance between treatment groups, when appropriate. Statistical differences were considered significant at the ${ }^{*} \mathrm{P}<0.05$ or ${ }^{* *} \mathrm{P}<0.01$ level.

\section{Results}

\section{S. aureus inhibits osteoblast proliferation}

In order to determine the effect of $S$. aureus, PVL, SpA and Coa on osteoblast survival or proliferation, MTT tetrazolium dye reduction assay was carried out. S. aureus were fixed to maintain bacterial cell integrity yet stunt their growth. This could prevent loss of essential nutrients necessary for osteoblast growth and proliferation. We investigated the ability of MC3T3-E1 cells to proliferate in the presence of $S$. aureus (or S. aureus overexpressing PVL, SpA or Coa or silencing PVL, SpA or Coa) or supernatant for $96 \mathrm{~h}$. Uninfected osteoblasts proliferated normally over the $96 \mathrm{~h}$ period. The osteoblasts growth slowed down by addition of the $S$. aureus supernatant or $S$. aureus silencing PVL, SpA or Coa. However, the difference did not reach significance (Fig. 1). Addition of S. aureus or S. aureus overexpressing PVL, SpA or Coa prevented osteoblasts from proliferation significantly compared to the uninfected osteoblasts $(\mathrm{P}<0.01)$ (Fig. 1). The proliferation inhibition of $S$. aureus overexpressing PVL, SpA or Coa group was even stronger than that of the $S$. aureus group.

\section{S. aureus induces osteoblast apoptosis}

Apoptosis was first determined by measuring the amount of FITC-Annexin V binding following incubation of MC3T3-E1 cells with formalin-treated S. aureus (or S. aureus overexpressing PVL, SpA or Coa or silencing PVL, SpA or Coa), or supernatant after a 24 $\mathrm{h}$ period. Apotosis was rarely found in the uninfected osteoblasts $(<5 \%)$. Compared to the uninfected osteoblasts, addition of $S$. aureus supernatant slightly increased the Annexin V binding $(<10 \%)$, while addition of $S$. aureus (or S. aureus overexpressing PVL, SpA or Coa or silencing PVL, SpA or Coa) induced apoptosis (Fig. 2A). The ratio of osteoblast apoptosis between the $S$. aureus and the supernatant group was statistically different (Fig. $2 \mathrm{~B}, \mathrm{P}<0.05$ ).

We next investigated the amount of pro-caspase 3, cleaved-caspase 3, pro-caspase 9 and cleaved-caspase 9 following incubation of MC3T3-E1 cells with formalin-treated S. aureus (or S. aureus overexpressing PVL, SpA or Coa or silencing PVL, SpA or Coa), or supernatant after a $24 \mathrm{~h}$ period. In the uninfected osteoblasts, caspase 3 cleavage and caspase 9 cleavage were minimal. No statistical difference of the pro-caspase 3, cleaved-caspase 3, pro-caspase 9 or cleaved-caspase 9 expression was found between the S. aureus silencing PVL, SpA or Coa 


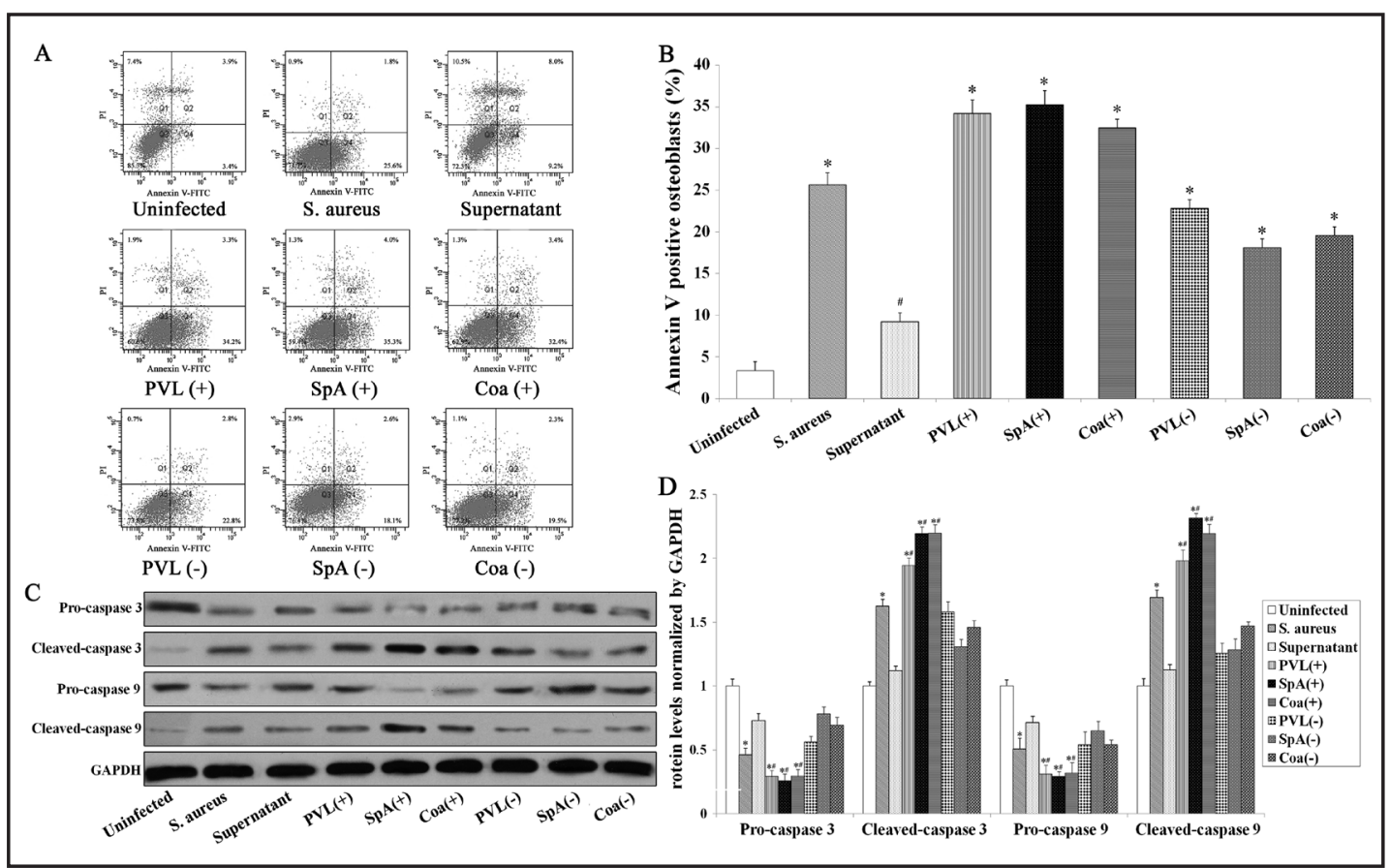

Fig. 2. S. aureus induces osteoblast apoptosis. MC3T3-E1 cells were treated with S. aureus (or S. aureus overexpressing PVL, SpA or Coa or silencing PVL, SpA or Coa), or supernatant for $24 \mathrm{~h}$. (A) To measure Annexin $\mathrm{V}$, treated osteoblasts were re-suspended in $100 \mu \mathrm{l}$ of FITC-Annexin V antibody. Suspensions were incubated in the dark for $15 \mathrm{~min}$ at RT and analysed by flow cytometry. (B) Quantification of the percentage of Annexin V positive MC3T3-E1 cells subjected to different treatments. (C) Western blot analysis of caspase 3 and caspase 9. (D) Quantitative analysis of caspase 3 and caspase 9 protein levels normalized by GAPDH. All values are the means \pm SD of three replicates, ${ }^{*} p<0.05$ vs uninfected, ${ }^{\#} \mathrm{p}<0.05$ vs $S$. aureus.

group and the uninfected osteoblasts. Similar situation was also found in the supernatant group. However, after incubation with $S$. aureus or S. aureus overexpressing PVL, SpA or Coa, the amount of pro-caspase 3 and pro-caspase 9 decreased significantly $(\mathrm{p}<0.05)$, while the amount of cleaved-caspase 3 and cleaved-caspase 9 increased significantly $(\mathrm{p}<0.05)$ (Fig. 2C, 2D). Compared to the $S$. aureus group, the amount of pro-caspase 3 and pro-caspase 9 decreased significantly $(\mathrm{p}<0.05)$, while the amount of cleaved-caspase 3 and cleavedcaspase 9 increased significantly $(\mathrm{p}<0.05)$ in the S. aureus overexpressing PVL, SpA or Coa group (Fig. 2C, 2D).

\section{S. aureus inhibits bone formation markers expression}

Osteoblasts secrete proteins including collagen I, osteopontin and osteocalcin to form extracellular matrix. To investigate the effect of S. aureus, PVL, SpA and Coa on the osteogenesis, the expression levels of collagen I, osteopontin and osteocalcin were detected by real time RT-PCR at $0 \mathrm{~d}, 7 \mathrm{~d}, 14 \mathrm{~d}$, and $21 \mathrm{~d}$. The gene expression was normalized to GAPDH expression and depicted as fold change $v s$ uninfected group at $0 \mathrm{~d}$. Collagen I is an early stage marker of osteogenesis that is typically expressed at day 7. The collagen I was increased about 2-fold in the uninfected osteoblasts at day 7. Compared to the uninfected MC3T3-E1, the expression of collagen I at day 7 was significantly lower in the MC3T3-E1 cells which were treated with $S$. aureus (or S. aureus overexpressing PVL, SpA or Coa or silencing PVL, SpA or Coa), or supernatant ( $\mathrm{p}<0.01$, Fig. 3A). The collagen I expression gradually decreased back to baseline at day 14 and 21 in the uninfected cells. There was no difference of collagen I expression in all the groups both at day 14 and day 21.

Osteopontin is a mid stage markers of osteogenesis. The osteopontin was increased about 18-fold in the uninfected osteoblasts at day 14. Compared to the uninfected group, 
Fig. 3. МС3Т3-E1 cells were treated with $S$. aureus (or $S$. aureus overexpressing $\mathrm{PVL}, \mathrm{SpA}$ or Coa or silencing PVL, SpA or Coa), or supernatant and incubated for 21 days. The mRNA expression of collagen I, osteopontin and osteocalcin was detected with RTPCR at $0 \mathrm{~d}, 7 \mathrm{~d}, 14 \mathrm{~d}$, and $21 \mathrm{~d}$. The mRNA expression of collagen I (A), osteopontin (B) and osteocalcin (C) was normalized to GAPDH expression and depicted as fold change vs Uninfected group at 0 d. All values are the means $\pm S D$ of three replicates, $" *<<0.01$ vs uninfected.

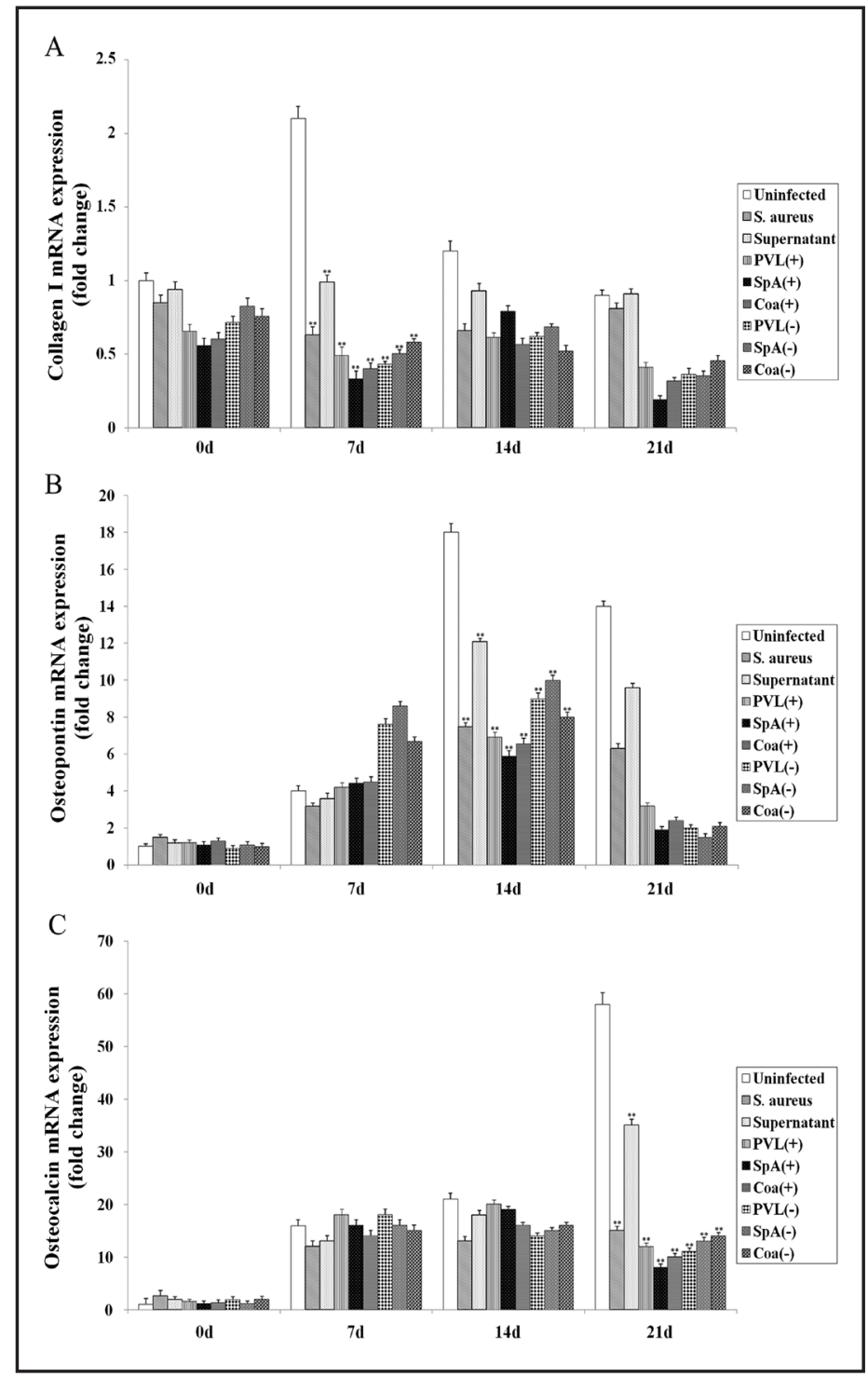

the expression of osteopontin at day 14 was significantly lower in the $S$. aureus (or $S$. aureus overexpressing PVL, SpA or Coa or silencing PVL, SpA or Coa), or supernatant group ( $\mathrm{p}<0.01$, Fig. 3B). Compared to the uninfected group, no statistical difference was found in the expression of osteopontin at day 7 in S. aureus (or S. aureus overexpressing PVL, SpA or Coa or silencing PVL, SpA or Coa), or supernatant group. Similar situation was also found at day 21.

Osteocalcin is a late stage marker of osteogenesis. Expression of osteocalcin was greatly increased at day 21 in the uninfected cells (at almost 60 -fold). Addition of $S$. aureus (or $S$. aureus overexpressing PVL, SpA or Coa or silencing PVL, SpA or Coa), or supernatant did not cause the great increase of osteocalcin expression at day 21 and resulted in significantly difference of osteocalcin expression between the uninfected group and the $S$. aureus (or $S$. aureus overexpressing PVL, SpA or Coa or silencing PVL, SpA or Coa), or supernatant group ( $p<0.01$, Fig. 3C). Compared to the uninfected group, no statistical difference was found in the expression of osteocalcin at day 7 in S. aureus (or S. aureus overexpressing PVL, SpA or Coa or silencing PVL, SpA or Coa), or supernatant group. Similar situation was also found at day 14. 


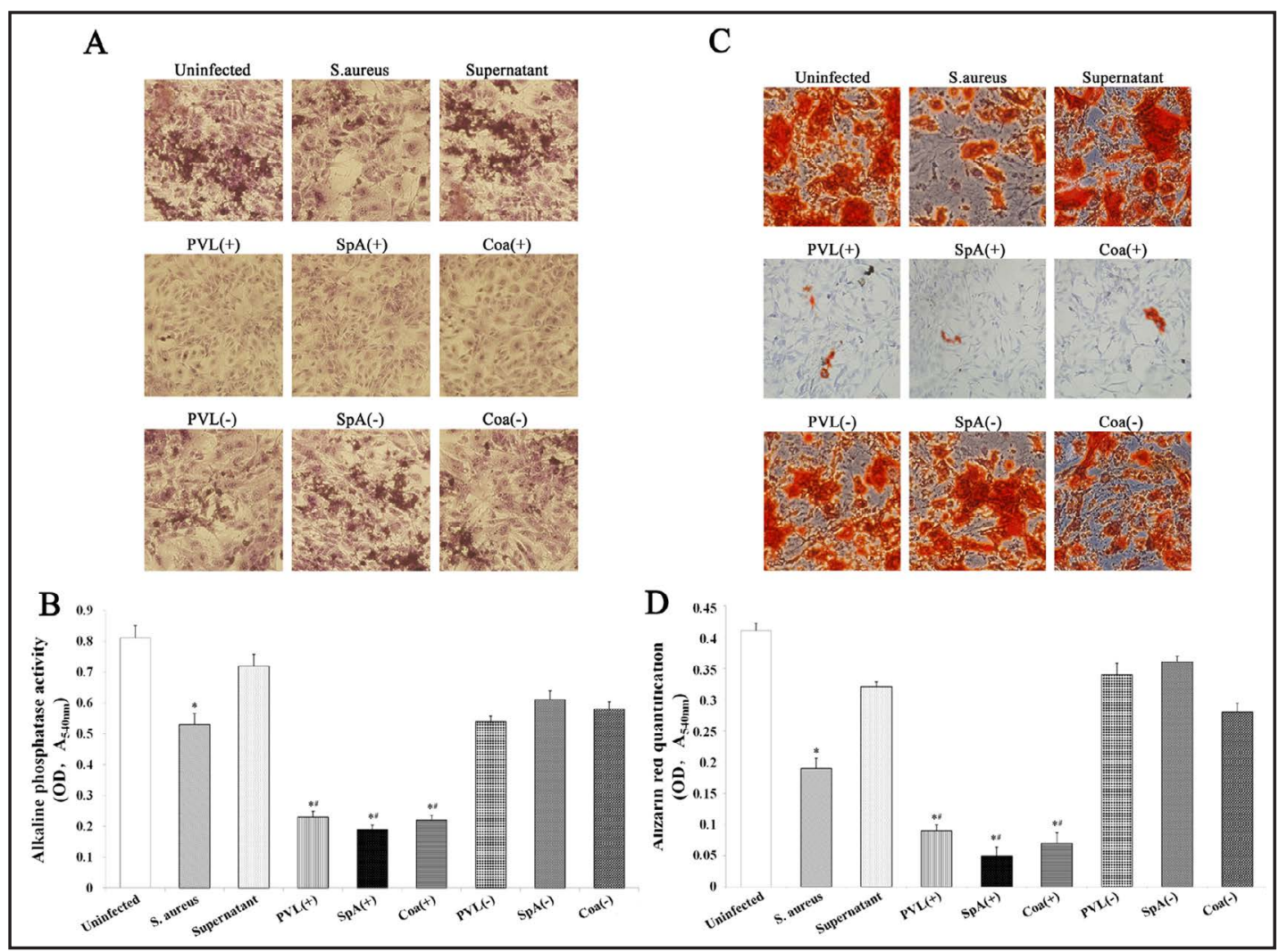

Fig. 4. S. aureus prevents osteoblast mineralisation. MC3T3-E1 cells were treated with S. aureus (or S. aureus overexpressing PVL, SpA or Coa or silencing PVL, SpA or Coa), or supernatant and incubated for 21 days. (A) At the 7 day infection, von kossa stain was added to the osteoblasts to determine phosphate deposition. (B) At the 7 day infection, osteoblasts were lysed with $1 \mathrm{ml}$ of lysis buffer containing a substrate for alkaline phosphatase (0.1 M Na acetate, $2 \%$ Triton X-100 and 10mM p-nitrophenol phosphate) and incubated in the dark for 1 hour at $37^{\circ} \mathrm{C}$, and reading absorbance at $540 \mathrm{~nm}$. (C) Following a 21 day infection, alizarin red stain (2\%) was added to the osteoblasts. (D) Staining was quantified by leeching the cells of the dye and reading absorbance at $540 \mathrm{~nm}$. Representative images for both stains were obtained using $200 \times$ bright field microscopy. All values are the means \pm SD of three replicates, ${ }^{*} p<0.05$ vs uninfected, ${ }^{\#} \mathrm{p}<0.05$ vs $S$. aureus.

\section{S. aureus prevents osteoblast mineralisation}

The calcification and mineralisation of bone matrix which facilitated by osteoblasts are essential for the strength and rigidity of the skeletal system. To estimate the osteoblastic mineralisation and calcification, staining for phosphates (von Kossa) at day 7 and for calcium deposition (Alizarin red) at day 21 were performed. Representative images of von kossa stain and alizarin red stain were obtained by brightfield microscopy and shown in Fig. 4A and 4C, respectively. Uninfected MC3T3-E1 showed signs of both phosphate and calcium deposition. Compared to the uninfected MC3T3-E1, the phosphate deposition of S. aureus overexpressing PVL, SpA or Coa was decreased greatly (Fig. 4A), the calcium deposition of $S$. aureus (S. aureus overexpressing PVL, SpA or Coa) was significantly decreased (Fig. 4C). Quantification of the calcium deposition (alizarin red staining) supported the results ( $\mathrm{p}<0.05$, Fig. 4D). What's more, compared to the $S$. aureus group, the calcium deposition was significantly decreased in the S. aureus overexpressing PVL, SpA or Coa group (Fig. 4D, $\mathrm{p}<0.05$ ).

ALP is an enzyme found in osteoblasts and pivotal for the bone mineralization. The ALP activity was determined in this study. Compared to the uninfected MC3T3-E1 cells, the ALP activity was decreased significantly in the $S$. aureus (S. aureus overexpressing PVL, SpA or 
Fig. 5. $S$. aureus induces RANK-L in osteoblasts. MC3T3-E1 cells were treated with $S$. aureus (or S. aureus overexpressing PVL, SpA or Coa or silencing PVL, SpA or Coa), or supernatant for $24 \mathrm{~h}$. Two hundred micro litres of the RIPA buffer was added to each well resulting protein was removed and centrifuged at $10,000 \mathrm{~g}$ for 2 minutes. RANK-L was detected using an ELISA kit. All values are the means \pm SD of three replicates, ${ }^{*} p<0.05$ vs uninfected, ${ }^{\#} \mathrm{p}<0.05$ vs S. aureus.

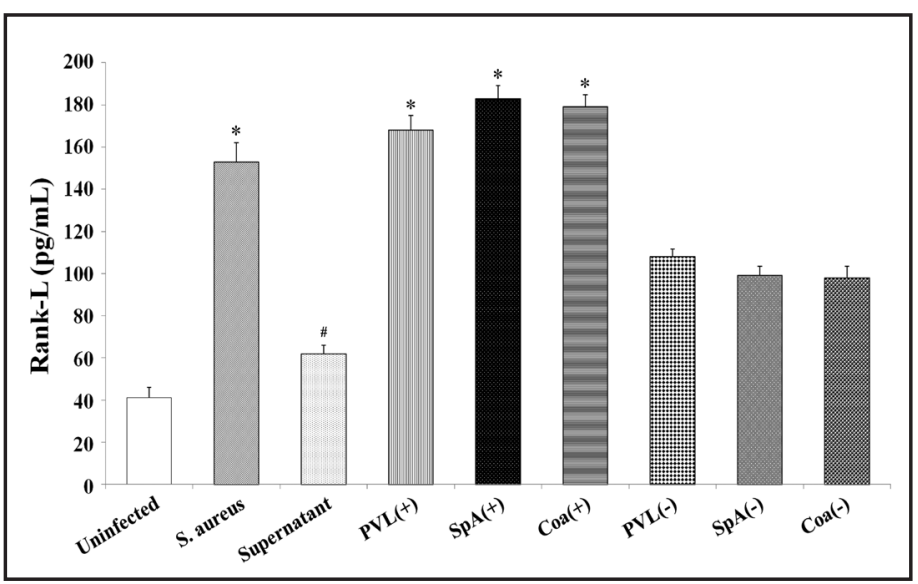

Coa) group ( $\mathrm{p}<0.05$, Fig. 4B). The ALP activity of S. aureus overexpressing PVL, SpA or Coa group was significantly lower than that of the $S$. aureus group ( $<<0.05$, Fig. $4 \mathrm{~B}$ ).

\section{S. aureus induces RANK-L in osteoblasts}

RANK-L which expressed on osteoblasts is a key molecule involved in bone remodeling. To investigate the effect of $S$. aureus (or S. aureus overexpressing PVL, SpA or Coa or silencing PVL, SpA or Coa), or supernatant on RANK-L expression, quantitative analysis was performed by ELISA. Uninfected MC3T3-E1 cells had a low level of RANK-L expression. The expression of RANK-L was increased in S. aureus (or S. aureus overexpressing PVL, SpA or Coa or silencing PVL, SpA or Coa), or supernatant group. And the difference between the uninfected group and the $S$. aureus or $S$. aureus overexpressing PVL, SpA or Coa group reached statistically significant $(\mathrm{p}<0.05$, Fig. 5$)$. The RANK-L expression of $S$. aureus group was significantly higher than that of supernatant group ( $\mathrm{p}<0.05$, Fig. 5 ).

\section{Discussion}

Osteomyelitis is a debilitating infectious disease of the bone which is predominantly caused by $S$. aureus. It is characterised by progressive bone loss or bone destruction. Currently the mechanism of bone loss or bone destruction in osteomyelitis is poorly understood. In this study, we investigated the role of three common $S$. aureus virulence factors including SpA, PVL and Coa on osteomyelitis.

$\mathrm{SpA}$ is a typical member of the microbial surface component recognizing adhesive matrix molecules (MSCRAMMs). It binds to a variety of ligands including the Fc region of IgG, von Willebrand factor (vWF), tumour necrosis factor receptor-1 (TNFR-1), the Fab-heavy chains of the Vh3 subclass and the epidermal growth factor receptor (EGFR) [33-37]. It is an important virulence factor of $S$. aureus for its mediation of colonization. PVL is a kind of exotoxin secreted by $S$. aureus. It is classified as a bicomponent cytolysin (LukF-PV and LukS-PV) and hetero-oligomerize to form a pore. PVL is cytotoxic toward leukocytes [38]. Coa is an enzyme secreted by $S$. aureus. It directly binds to prothrombin and leads to plasma clot [39]. The effect of SpA, PVL and Coa on osteoblasts was studied through the following aspects including osteoblast proliferation, apoptosis, bone formation, bone mineralization and RANK-L expression. S. aureus overexpressing PVL, SpA or Coa was constructed and used to study the role of PVL, SpA and Coa, respectively. S. aureus silencing PVL, SpA or Coa was also constructed and used for reversing verification.

Our study demonstrated that $S$. aureus inhibited osteoblast proliferation and induced osteoblast apotosis. The proliferation inhibition was aggravated by $S$. aureus overexpressing PVL, SpA or Coa and the proliferation was almost unaffected by $S$. aureus silencing PVL, SpA or Coa. The results indicated that SpA, PVL and Coa might trigger a signal transduction 
pathway that inhibits osteoblast proliferation. Significant apoptosis was observed in both S. aureus overexpressing PVL, SpA or Coa group and S. aureus silencing PVL, SpA or Coa group. The apoptosis was more serious in the presence of PVL, SpA or Coa. These results suggested that other S. aureus proteins besides PVL, SpA and Coa might induce apoptosis. Further experiment showed that the cleaved-caspase 3 and cleaved-caspase 9 expressed highly in S. aureus overexpressing PVL, SpA or Coa group. Caspase-9 is an initiator caspase and links to the mitochondrial death pathway of apoptosis [40]. It interacts with caspase 3. The activation of both caspase 3 and caspase 9 in the presence of PVL, SpA or Coa indicating that PVL, SpA or Coa triggered mitochondrial death pathway.

Bone formation is typically characterised by the sequential expression of a series of bone formation markers including collagen I, osteopontin and osteocalcin. The expression of collagen I, osteopontin and osteocalcin were decreased significantly in both S. aureus overexpressing PVL, SpA or Coa group and S. aureus silencing PVL, SpA or Coa group in this study. The results suggested that bone formation was disrupted in these situations. Other proteins beside PVL, SpA or Coa may inhibit the osteogenesis. Mineralisation is a process where phosphate and calcium becomes deposited in bone [41]. This gives the bones additional strength and rigidity. Our study showed that mineralization (phosphate and calcium deposition) was inhibited significantly in the $S$. aureus overexpressing PVL, SpA or Coa group, while phosphate and calcium deposition formed normally in $S$. aureus silencing PVL, SpA or Coa group. Besides, the ALP activity was also decreased significantly in the S. aureus (S. aureus overexpressing PVL, SpA or Coa) group. These indicated that bone mineralization had been inhibited by PVL, SpA or Coa.

RANK-L is a membrane protein of osteoblasts. It stimulates differentiation in osteoclasts and involves in bone resorption [42]. A significant increase in RANK-L expression was found in $S$. aureus overexpressing PVL, SpA or Coa group, The increase in RANKL can trigger osteoclast-induced bone resorption and bone destruction. This may also lead to bone loss in osteomyelitis. Furthermore, S. aureus silencing PVL, SpA or Coa had no affect on the RANK-L expression, which revealed that PVL, SpA and Coa were the important virulence factors of $S$. aureus in osteomyelitis.

In summary, we evaluated the role of PVL, SpA or Coa on osteomyelitis by constructing S. aureus overexpressing PVL, SpA or Coa and S. aureus silencing PVL, SpA or Coa. PVL, SpA and Coa inhibited osteoblast proliferation, induced osteoblast apotosis, prohibited bone formation and mineralization and triggered osteoclast-induced bone resorption and bone destruction by upregulated RANK-L expression. PVL, SpA and Coa play a critical role on bone destruction and bone loss of osteomyelitis.

\section{Conflict of Interests}

The authors stated that they had no interests which might be perceived as posing a conflict or bias.

\section{Acknowledgements}

The study was supported by National Natural Science Foundation of China (No: 81171734).

\section{References}

1 Martin TJ, Sims NA: Osteoclast-derived activity in the coupling of bone formation to resorption. Trends Mol Med 2005;11:76-81.

2 Millan JL: The role of phosphatases in the initiation of skeletal mineralization. Calcif Tissue Int 2013, in press. 
3 Niu LN, Jiao K, Ryou H, Yiu CK, Chen JH, Breschi L, Arola DD, Pashley DH, Tay FR: Multiphase intrafibrillar mineralization of collagen. Angew Chem Int Ed Engl 2013;52:5762-5766.

-4 Bondarenko A, Angrisani N, Meyer-Lindenberg A, Seitz JM, Waizy H, Reifenrath J: Magnesium-based bone implants: Immunohistochemical analysis of peri-implant osteogenesis by evaluation of osteopontin and osteocalcin expression. J Biomed Mater Res A 2013; doi: 10.1002/jbm.a.34828.

5 Ribeiro N, Sousa SR, Monteiro FJ: Influence of crystallite size of nanophased hydroxyapatite on fibronectin and osteonectin adsorption and on mc3t3-e1 osteoblast adhesion and morphology. J Colloid Interface Sci 2010;351:398-406.

6 Nakashima T, Hayashi M, Fukunaga T, Kurata K, Oh-Hora M, Feng JQ, Bonewald LF, Kodama T, Wutz A, Wagner EF, Penninger JM, Takayanagi H: Evidence for osteocyte regulation of bone homeostasis through rankl expression. Nat Med 2011;17:1231-1234.

7 Garzoni C, Kelley WL: Staphylococcus aureus: New evidence for intracellular persistence. Trends Microbiol 2009;17:59-65.

8 Lew DP, Waldvogel FA: Osteomyelitis. Lancet 2004;364:369-379.

-9 Klosterhalfen B, Peters KM, Tons C, Hauptmann S, Klein CL, Kirkpatrick CJ: Local and systemic inflammatory mediator release in patients with acute and chronic posttraumatic osteomyelitis. J Trauma 1996;40:372-378.

10 Marriott I, Gray DL, Tranguch SL, Fowler VG Jr, Stryjewski M, Scott Levin L, Hudson MC, Bost KL: Osteoblasts express the inflammatory cytokine interleukin-6 in a murine model of staphylococcus aureus osteomyelitis and infected human bone tissue. Am J Pathol 2004;164:1399-1406.

-11 Carek PJ, Dickerson LM, Sack JL: Diagnosis and management of osteomyelitis. Am Fam Physician 2001;63:2413-2420.

12 Dhanoa A, Singh VA, Mansor A, Yusof MY, Lim KT, Thong KL: Acute haematogenous community-acquired methicillin-resistant staphylococcus aureus osteomyelitis in an adult: Case report and review of literature. BMC Infect Dis 2012;12:270.

13 Rao N, Ziran BH, Lipsky BA: Treating osteomyelitis: Antibiotics and surgery. Plast Reconstr Surg 2011;127:177S-187S.

14 Zuluaga AF, Galvis W, Saldarriaga JG, Agudelo M, Salazar BE, Vesga O: Etiologic diagnosis of chronic osteomyelitis: A prospective study. Arch Intern Med 2006;166:95-100.

15 Wright JA, Nair SP: Interaction of staphylococci with bone. Int J Med Microbiol 2010;300:193-204.

16 DeLeo FR, Otto M, Kreiswirth BN, Chambers HF: Community-associated meticillin-resistant staphylococcus aureus. Lancet 2010;375:1557-1568.

17 Tenover FC, Goering RV: Methicillin-resistant staphylococcus aureus strain usa300: Origin and epidemiology. J Antimicrob Chemother 2009;64:441-446.

18 Foster TJ: Colonization and infection of the human host by staphylococci: Adhesion, survival and immune evasion. Vet Dermatol 2009;20:456-470.

19 Jarraud S, Mougel C, Thioulouse J, Lina G, Meugnier H, Forey F, Nesme X, Etienne J, Vandenesch F: Relationships between staphylococcus aureus genetic background, virulence factors, agr groups (alleles), and human disease. Infect Immun 2002;70:631-641.

-20 Begun J, Sifri CD, Goldman S, Calderwood SB, Ausubel FM: Staphylococcus aureus virulence factors identified by using a high-throughput caenorhabditis elegans-killing model. Infect Immun 2005;73:872877.

21 Zecconi A, Scali F: Staphylococcus aureus virulence factors in evasion from innate immune defenses in human and animal diseases. Immunol Lett 2013;150:12-22.

22 Lofblom J, Kronqvist N, Uhlen M, Stahl S, Wernerus H: Optimization of electroporation-mediated transformation: Staphylococcus carnosus as model organism. J Appl Microbiol 2007;102:736-747.

23 Stoll H, Dengjel J, Nerz C, Gotz F: Staphylococcus aureus deficient in lipidation of prelipoproteins is attenuated in growth and immune activation. Infect Immun 2005;73:2411-2423.

24 Merino N, Toledo-Arana A, Vergara-Irigaray M, Valle J, Solano C, Calvo E, Lopez JA, Foster TJ, Penades JR, Lasa I: Protein a-mediated multicellular behavior in staphylococcus aureus. J Bacteriol 2009;191:832-843.

25 Arnaud M, Chastanet A, Debarbouille M: New vector for efficient allelic replacement in naturally nontransformable, low-gc-content, gram-positive bacteria. Appl Environ Microbiol 2004;70:6887-6891. 


\section{Cellular Physiology Cell Physiol Biochem 2013;32:322-333 \begin{tabular}{l|l} 
DOI: $10.1159 / 000354440$ & (c) 2013 S. Karger AG, Basel
\end{tabular} \\ Jin et al.: Virulence Factors of $S$. Aureus Aggravate Osteomyelitis}

26 Murphy CM, Haugh MG, O'Brien FJ: The effect of mean pore size on cell attachment, proliferation and migration in collagen-glycosaminoglycan scaffolds for bone tissue engineering. Biomaterials 2010;31:461466.

27 Plunkett NA, Partap S, O'Brien FJ: Osteoblast response to rest periods during bioreactor culture of collagen-glycosaminoglycan scaffolds. Tissue Eng Part A 2010;16:943-951.

-28 Tuchscherr L, Medina E, Hussain M, Volker W, Heitmann V, Niemann S, Holzinger D, Roth J, Proctor RA, Becker K, Peters G, Loffler B: Staphylococcus aureus phenotype switching: An effective bacterial strategy to escape host immune response and establish a chronic infection. EMBO Mol Med 2011;3:129-141.

29 Tuchscherr L, Heitmann V, Hussain M, Viemann D, Roth J, von Eiff C, Peters G, Becker K, Loffler B: Staphylococcus aureus small-colony variants are adapted phenotypes for intracellular persistence. J Infect Dis 2010;202:1031-1040.

-30 Zhang D, Jiang P, Xu Q, Zhang X: Arginine and glutamate-rich 1 (arglu1) interacts with mediator subunit 1 (med1) and is required for estrogen receptor-mediated gene transcription and breast cancer cell growth. J Biol Chem 2011;286:17746-17754.

-31 Tsao FH, Xiang Z, Abbasi A, Meyer KC: Neutrophil necrosis and annexin 1 degradation associated with airway inflammation in lung transplant recipients with cystic fibrosis. BMC Pulm Med 2012;12:44.

32 Tong X, Lin S, Fujii M, Hou DX: Echinocystic acid induces apoptosis in hl-60 cells through mitochondriamediated death pathway. Cancer Lett 2004;212:21-32.

-33 Cedergren L, Andersson R, Jansson B, Uhlen M, Nilsson B: Mutational analysis of the interaction between staphylococcal protein a and human igg1. Protein Eng 1993;6:441-448.

-34 O'Seaghdha M, van Schooten CJ, Kerrigan SW, Emsley J, Silverman GJ, Cox D, Lenting PJ, Foster TJ: Staphylococcus aureus protein a binding to von willebrand factor a1 domain is mediated by conserved igg binding regions. FEBS J 2006;273:4831-4841.

-35 Gomez MI, O'Seaghdha M, Magargee M, Foster TJ, Prince AS: Staphylococcus aureus protein a activates tnfr1 signaling through conserved igg binding domains. J Biol Chem 2006;281:20190-20196.

36 Viau M, Zouali M: Effect of the b cell superantigen protein a from s. Aureus on the early lupus disease of (nzbxnzw) f1 mice. Mol Immunol 2005;42:849-855.

37 Gomez MI, Seaghdha MO, Prince AS: Staphylococcus aureus protein a activates tace through egfrdependent signaling. EMBO J 2007;26:701-709.

-38 Kaneko J, Kamio Y: Bacterial two-component and hetero-heptameric pore-forming cytolytic toxins: Structures, pore-forming mechanism, and organization of the genes. Biosci Biotechnol Biochem 2004;68:981-1003.

-39 Vanassche T, Verhaegen J, Peetermans WE, Hoylaerts MF, Verhamme P: Dabigatran inhibits staphylococcus aureus coagulase activity. J Clin Microbiol 2010;48:4248-4250.

40 MacKenzie SH, Clark AC: Death by caspase dimerization. Adv Exp Med Biol 2012;747:55-73.

41 Hill PA: Bone remodelling. Br J Orthod 1998;25:101-107.

42 Boyce BF, Xing L: Functions of rankl/rank/opg in bone modeling and remodeling. Arch Biochem Biophys 2008;473:139-146. 
The original article by Jin et al., entitled "Staphylococcal Protein A, Panton-Valentine Leukocidin and Coagulase Aggravate the Bone Loss and Bone Destruction in Osteomyelitis" [Cell Physiol Biochem 2013;32:322-333 (DOI: 10.1159/000354440)], failed to discuss and cite previous work and to acknowledge the original authors for identifying.

The authors wish to point out that Staphylococcus aureus protein A has previously been shown to interact with osteoblasts. As a result of this interaction osteoblasts fail to proliferate, mineralize or express critical osteogenic markers collagen type I, osteopontin or osteocalcin when engaged with protein A. In addition, the interaction resulted in apoptosis and increased recruitment of the bone resorbing cells, osteoclasts. These interactions were identified by another group and published in 2011 and 2012 (1,2, respectively). The authors of the current article failed to discuss or cite this previous work and acknowledge that due credit must go to the original authors for identifying this interaction.

\section{Reference}

1 Claro T, Widaa A, O’Seaghdha M, Miajlovic H, Foster TJ, O’Brien FJ, Kerrigan SW: Staphylococcus aureus protein A binds to osteoblasts and triggers signals that weaken bone in osteomyelitis. PLoS ONE 201115;6:e18748.

2 Widaa A, Claro T, Foster TJ, O’Brien FJ, Kerrigan SW: Staphylococcus aureus Protein A plays a critical role in mediating bone destruction and bone loss in osteomyelitis. PLoS ONE 2012;7:e40586. 